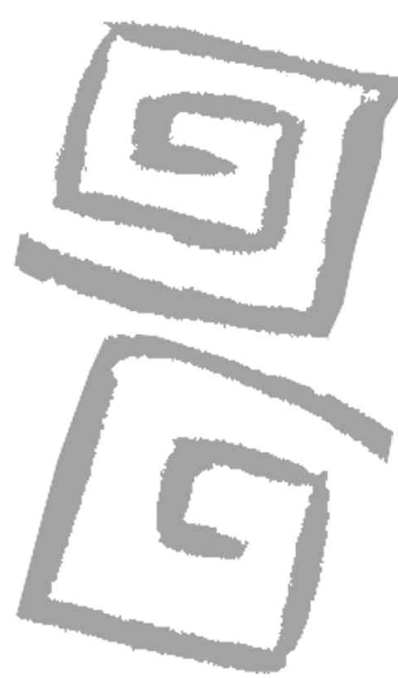

\title{
Contribuciones metodológicas para estudiar la producción del cuidado en salud: aprendizajes a partir de una investigación sobre barreras y acceso en salud mental
}

\author{
Methodological contributions towards the study of \\ health care production: lessons from a research \\ study on barriers and access in mental health
}

Merhy, Emerson Elias'; Feuerwerker, Laura Camargo Macruz²; Silva, Erminia ${ }^{3}$

\footnotetext{
${ }^{1}$ Doctor en Salud Colectiva. Profesor Titular, Universidade Federal do Rio de Janeiro (UFRJ), Brasil. emerson.merhy@gmail.com

${ }^{2}$ Médica. Doctora en Salud Pública. Profesora,

Universidade de São Paulo (USP). Investigadora, Departamento de Clínica Médica, Universidade Federal do Rio de Janeiro (UFRJ), Brasil. laura.macruz@gmail.com

${ }^{3}$ Historiadora. Doctora en Historia de la Cultura. Profesora invitada, posgrado del Instituto de Arte, Universidade Estadual de São Paulo (UNESP).

mina.silva@gmail.com
}

RESUMEN Este artículo presenta algunas contribuciones metodológicas y una innovación conceptual para pensar la producción del cuidado en salud, a partir de una investigación sobre el acceso y las barreras en salud mental realizada en la municipalidad de Campinas (San Pablo, Brasil). La investigación utilizó el abordaje cartográfico y, a partir de una identificación inicial de los casos más complejos (por parte de los equipos de trabajadores), adoptó a los usuarios como guías para recorrer los diferentes planos de producción de sus vidas y evaluar la posibilidad de armar, o no, una red de conexiones existenciales productoras de vida como analizador fundamental del acceso o barrera al cuidado.

PALABRAS CLAVE Accesibilidad a los Servicios de Salud; Salud Mental; Cartografía.

\begin{abstract}
This article presents methodological contributions and a conceptual innovation for thinking about the production of health care, stemming from a study on access and barriers in mental health carried out in the municipality of Campinas (São Paulo, Brazil). The study used a cartographic approach and, after an initial identification of the most complex cases (on the part of the teams of workers), adopted the users as guides to explore the different levels of production of their lives and to evaluate the possibility of forming a network of existential connections that produce life as a fundamental analyzer of access or barriers to care.
\end{abstract}

KEY WORDS Health Services Accessibility; Mental Health; Cartography. 


\section{APUESTAS FUNDAMENTALES}

Dos apuestas fundamentales han orientado este trabajo, en términos de su construcción en el campo de los estudios cartográficos, como los entienden Deleuze y Guattari en el capítulo "Rizoma" del libro Mil Mesetas (1).

La primera es la apuesta a la producción de diferentes sentidos en aquellos procesos de evaluación en los que los investigadores y los investigados son los mismos. Para esto es necesario trabajar la implicación y los afectos como constitutivos del campo de investigación y de la producción del conocimiento. Este proceso focaliza sobre la confrontación entre lo que es evaluado y lo que se produce como una doble implicación, ya que el objeto de conocimiento es el propio hacer del investigador que, como tal, se confronta consigo mismo. Esa característica implicó exponer a los trabajadores/investigadores a procesos de educación permanente (o sea, a analizar su propio quehacer) como modo de evaluar en acto e intervenir en acto sobre su trabajo y sobre sí mismos. La investigación, entonces, opera como dispositivo de subjetivación sobre los cuerpos colectivos de acción en el mundo del trabajo y como proceso de recolección de los saberes así producidos.

La otra apuesta se expresó en la pretensión de escapar a la clásica construcción de indicadores habituales de los estudios evaluativos. Exploramos la posibilidad de identificar no solo las barreras que pudieran ser captadas por indicadores propuestos externamente, sino también las barreras y definiciones de acceso creadas por los propios constructores de las redes de cuidado -trabajadores y usuarios- a partir de sus perspectivas. El equipo decidió construir algoritmos que nos permitieran analizar la producción del cuidado en salud como modos singulares de construcción de redes de conexiones existenciales, los que terminaron siendo nuestros principales marcadores de sentido.

Esta noción de redes no es de aprehensión inmediata, por lo que merece que se explicite su construcción. Las redes son el producto del diálogo entre la micropolítica del trabajo vivo y las apuestas de producción de cuidado en salud, en la defensa intransigente de la vida (2), con ciertas experiencias vividas en el campo de las prácticas de las luchas antimanicomiales (3).
De un modo didáctico, la mejor forma de entenderla es a través de lo que conocemos del cuidado manicomial: aísla, disciplinariza moralmente y medicamentaliza a aquel que considera un "enfermo mental". En ese proceso, corta las múltiples redes de vínculos existenciales existentes y ofrece como única posibilidad otra red de conexión, que se presenta como más protectora y controlada.

Entendemos que con esto "condena" a alguien a vivir exclusivamente bajo redes de conexiones definidas a partir de la enfermedad como centro de la producción de una vida cualquiera y se prohíben otras formas de vivir que no estén generadas a partir de ese territorio existencial. En esa dirección, Franco Basaglia (4) decía que debíamos colocar la enfermedad entre paréntesis para que en las relaciones de producción de vida emerjan otras vidas posibles que no partieran de aquella condena de existir solo a partir de la enfermedad diagnosticada. Por eso, hablaba de la necesidad de hacer una reforma psiquiátrica en el campo del cuidado al loco.

Ese camino, allí en el cotidiano del cuidado en salud, significa para nosotros que la producción de redes de conexiones existenciales, al ser dirigida -e incluso ampliada- por una única posibilidad de ordenamiento, como en el caso de la vida enferma, no expresaría la producción de más vida y, como tal, sería visto por nosotros como barrera, por impedir nuevos sentidos de y en el vivir.

Desde esta perspectiva concebimos la producción de nuevas redes de conexiones existenciales, como se verá en la descripción del estudio, y cómo esto está unido a un posicionamiento de un cuidado en defensa de la vida individual y colectiva, del cual nos interesa el enriquecimiento de las formas de vida, y cuán articulado está al campo de producción tecnológica en salud del trabajo vivo en acto (5).

Para lograr esto, debimos innovar en los abordajes de investigación, pues queríamos aproximarnos al movimiento de los usuarios que, en sus nomadismos y encuentros, construyen redes de conexiones existenciales. También queríamos aproximarnos al movimiento de los trabajadores, que en la micropolítica de sus encuentros operan dispositivos que inhiben o facilitan esas conexiones. 
Esto requirió, desde el diseño de la investigación, la construcción de un actuar colectivo con trabajadores de las redes instituidas de cuidado en salud mental, presuponiendo la micropolítica del trabajo vivo en acto como terreno de su producción.

Entonces, fue fundamental construir entre todos una ética de las relaciones necesarias para soportar un estudio tan delicado. Trabajar la ética de la producción del conocimiento, como tensión constitutiva entre el cuidar de sí y el conocerse a sí, demanda un gran esfuerzo para establecer que lo que hay de distinto en el otro me interesa, pero al mismo tiempo debe quedar claro que la intención no es colocarlo bajo análisis sin que el propio investigador se exponga al mismo proceso analítico.

Una vez más, operar en ese territorio en el flujo del trabajo vivo en acto requería una caja de herramientas que mezclara todo lo que estuviera disponible y tuviera sentido. Comprendimos que el camino de la investigación cartográfica presentaba esa ventaja: colocaba en acto, en actualización, todos los ejes de fuerzas de los diversos regímenes de verdad que buscan emerger en el territorio micropolítico.

\section{LA CONSTRUCCIÓN DE LA INVESTIGACIÓN Y SUS DEVENIRES}

Propusimos Campinas (San Pablo) como campo para analizar el tema del acceso y las barreras en salud mental, por ser un municipio que cuenta con una extensa y rica red de atención en el área de salud. Esta red fue pionera en la producción de determinados dispositivos terapéuticos orientados por los principios de la defensa de la vida. Por su calificación y sofisticación, esta red nos pareció un excelente escenario para analizar las barreras y el acceso, ya que en otros casos podríamos estar analizando situaciones obvias determinadas por la falta de oferta.

Este artículo es el producto de esta investigación desarrollada durante los años 2008 y 2011, titulada Acesso e barreira em uma complexa rede de cuidado: o caso de Campinas, realizada a partir de una convocatoria conjunta del Ministerio de Salud y del Ministerio de Ciencia y
Tecnología, por intermedio del Consejo Nacional de Desarrollo Científico y Tecnológico (CNPq) de Brasil. El equipo de investigación estuvo compuesto por participantes de la Línea de Micropolítica del Trabajo y del Cuidado en Salud (Universidade Federal do Rio de Janeiro) y por trabajadores de la red de atención de salud mental del Servicio de Salud Dr. Cândido Ferreira, de Campinas, Brasil. El proyecto fue aprobado por el Comité de Ética en Investigación del Hospital Universitário Clementino Fraga Filho de la Universidade Federal do Rio de Janeiro.

\section{Conceptos importantes en la construcción metodológica}

Hay estudios en el campo del cuidado en salud que entienden la idea del territorio como algo físico, rígido y geográfico. En realidad, los individuos, en tanto usuarios de los establecimientos de salud, buscan generalmente formas de vínculos relacionales con diversos territorios posibles de un modo muy singular, tomando esa construcción como algo subjetivante. El concepto de territorio es, entonces, existencial y situacional, y tiene muchos sentidos.

De esta manera, los usuarios toman para sí, por un lado, el territorio geográfico socialmente constituido como un referencial que para ellos tiene sentido. Así, su barrio y todo lo que existe en él, en términos de equipamientos institucionales, son referencias subjetivas por estar en el campo de sentido territorial. Por otro lado, esa dimensión en sí es también cultural y, como tal, sobrepasa lo geográfico, de tal manera que los usuarios fabrican otra geografía: buscan además otras ofertas que consideran más adecuadas, situadas en otros sitios que no son los de su barrio o de su lugar de trabajo $(2,6)$.

En el campo del cuidado en salud mental (7), esa segunda situación es muy significativa. El usuario es un "nómade" por las redes de cuidado, siendo incluso un fuerte protagonista de la producción de su propio cuidado $(8,9)$.

De allí surge un problema metodológico: situarse en los establecimientos de salud como lugar central para construir las fuentes de información no permite visibilizar el cuidado producido en toda su complejidad, la cual corre 
por los flujos existenciales que posibilitan los múltiples procesos relacionales construidos por los usuarios en sus modos de andar la vida (10).

De ese modo, todos los usuarios de las redes de salud, aunque estén fuertemente vinculados a ciertos equipos de salud, no son sus usuarios exclusivos, a pesar de existir una fuerte territorialización. Consumen y producen otros lazos. Son "nómades" en el sentido de que son productores de redes de conexiones existenciales no previstas y conocidas en el mundo del cuidado; redes de conexiones que escapan a los lugares instituidos como propios por los servicios de salud.

Por lo tanto, nuestra cartografía no podría restringirse a la construcción de itinerarios terapéuticos. Era necesario caminar con los usuarios y descubrir la producción, muchas veces en acto, de nuevas redes de conexiones. Entonces, todas las "estaciones de cuidado" serían fuentes para revelar el acceso y las barreras, pero no serían suficientes (11).

En otras palabras, el usuario en general tiene un comportamiento "nómade" por las redes de conexión del cuidado, pues no utiliza solo los equipos de salud para obtener cuidado; produce otras y nuevas redes-acontecimientos, desterritorializadas, con su modo "nómade" de andar la vida (8).

Así, las estaciones de cuidado eran fuentes iniciales importantes, pero resultaba imprescindible cruzar múltiples estrategias de investigación, capaces de operar los diversos regímenes de verdades que emergen, como por ejemplo, los producidos en los intensos procesos subjetivantes constituidos por la micropolítica de los encuentros imprevisibles, constitutivos de los diversos modos de andar la vida.

Debido a las características del propio campo de la salud y del modo en que se producen los modos de vivir, sería necesario explorar el nomadismo singular de cada usuario, tomándolo como caso-guía, para percibir cómo construyen nuevas redes de cuidado. Estas redes de cuidado pueden existir incluso fuera del sistema de salud, y son construidas por el usuario, muchas veces, con el propósito de vencer las barreras al cuidado. En otras ocasiones, estas redes fuera del sistema de salud se construyen por encuentros no previstos.
Por eso es que incorporamos a la perspectiva metodológica de Merhy y Feuerwerker, ya utilizada en otras investigaciones $(12,13)$, la metodología que la historiadora Ermínia Silva (14) utilizó en su investigación de la historia social de la cultura del circo-teatro.

En tal investigación, para enfrentar el nomadismo de los artistas circenses y del mismo circo, la investigadora trabajó con la trayectoria de algunos artistas como guías que le han permitido mapear lugares, instituciones, prácticas y saberes. Así, pudo cartografiar redes con múltiples conexiones que le abrieron el acceso a diversas fuentes que le brindaron elementos de construcción de la memoria de el/los modo/s de vivir/es circenses, en la marcha nómade, típica de ese grupo social.

En el estudio sobre salud mental, los equipos han sido las fuentes iniciales para llegar a los casos "usuario-guía" que permitieran mapear y analizar mejor, en una rica red sustitutiva con gran multiplicidad de ofertas, las barreras y accesibilidades al cuidado en salud mental.

Dado que la producción social de salud se realiza mediante el trabajo vivo en acto, la construcción del conocimiento sobre este trabajo exige ir más allá de la sencilla relación sujeto/objeto. Esta característica del trabajo vivo en acto, que no puede ser registrada plenamente, requiere que los propios investigadores estén directamente involucrados en esa producción para que proporcionen, aunque de modo fragmentario, ciertas informaciones. Además, sustancialmente, como se trata de un proceso con implicancias narrativas, se necesita trabajar incesantemente en la búsqueda de otras fuentes, de distinta naturaleza, que en su multiplicidad permitan analizar, por intermedio de sus alteridades (5) el proceso de construcción de ciertos regímenes de verdad, como dicen Foucault y Deleuze $(15,16)$.

\section{LOS PASOS DE LA INVESTIGACIÓN}

Uno de los primeros debates estuvo vinculado a la habitual práctica del campo de la investigación en salud de analizar a los usuarios tomando como referente el diagnóstico de su 
enfermedad. El equipo discutió que si se partía solo de los diagnósticos, se produciría un significativo desvío respecto a nuestras pretensiones, pues esos registros son producidos, en muchos casos, a partir de concepciones que toman los sufrimientos mentales bajo la óptica de la enfermedad, o sea, de un modo muy limitado. Los límites e implicancias de esa perspectiva, de ese régimen de verdad, han sido ya identificados en estudios muy provocadores y reveladores, como los de Foucault sobre la emergencia de la locura y su construcción como objeto de la medicina psiquiátrica (17). Adoptar esa mirada implicaría someternos a limitaciones, pues los usuarios intensivos de servicios, que demandan cuidados en múltiples modalidades, no necesariamente son los que tienen los diagnósticos más dramáticos desde el punto de vista médico; son otros los elementos que crean esa complejidad y gravedad. Además, los diagnósticos se resumen en un código de la Clasificación Internacional de Enfermedades, y se constituyen en una marca que tiende a restringir más que a ampliar, limitando las posibilidades de aquellos investigadores que buscan la producción de redes de conexiones existenciales. Los usuarios, con sus complejas vidas e implicaciones, van mucho más allá de lo que los diagnósticos permiten entrever, incluso como referencia para la producción del cuidado. Aún menos para una investigación como la que nos propusimos realizar.

Empezamos, entonces, con las definiciones que produjo el grupo de trabajadores del área de salud mental de Campinas sobre los que serían "los locos muy locos", es decir, aquellos que demandaban los servicios de los equipos de salud con mayor intensidad, en el cotidiano de la producción del cuidado. Estos "locos muy locos" eran los que exigían con su acción, la máxima capacidad de crear lógicas intercesoras de encuentros, independientemente del diagnóstico que portaban (2).

De este modo, esperábamos obtener, como material de estudio, los modos de producir cierta atención a la locura, colocando en escena la tensión acceso/barrera como construcción cotidiana del cuidar y no necesariamente como pasaje físico de un lugar situado afuera hacia otro lugar dentro del servicio de salud, o desde un nivel de cuidado hacia otro, como línea de acción en el campo de los servicios.
Cada equipo -de los distintos tipos de servicios que componen la red de cuidados en salud mental- eligió un caso. Los casos fueron extensamente reconstruidos en el colectivo, a partir de las memorias de los mismos equipos. En un primer momento, el colectivo produjo trayectorias de los flujogramas analizadores, mapas de los territorios existenciales, creando cierto proceso cartográfico con cada una de las indicaciones. Todo esto se hizo de manera provisoria, solo con el sentido de la experimentación: fabricando marcadores y referenciales que nos permitieran movernos en aquellos territorios de conexiones.

Uno de los casos, el "caso Branca", fue paradigmático para el desarrollo de los diseños de la investigación cartográfica, a partir del cual conformamos la noción que adoptaríamos posteriormente referente a la construcción de "redes de conexiones existenciales". Con este caso, percibimos la necesidad de cruzar la "historia de vida", con la "historia de los núcleos familiares", la "historia en el territorio de la vida común", la "historia de las enfermedades", la producción del "mapa de lo instituido en las conexiones con los servicios de salud", y la necesidad de destacar en este mapeo el recorte de los servicios del campo del cuidado en salud mental.

Además, percibimos que había muchas "Brancas" circulando en este estudio, definidas de modo situacional según las conexiones que se producían. Comprendimos la necesidad de seguir las narrativas que pudieran iluminar esas múltiples producciones y cruzarlas, hacerlas conversar y buscar interpretarlas. Asimismo, comprendimos que debíamos explorar todas las posibilidades de construcción narrativa de los diversos regímenes de verdad que operaban en nuestra producción, en los "textos" que encontramos con la exploración y en el estudio de las fuentes registradas y orales que cada "caso" nos abría.

Comprendimos claramente que la "historia de vida" es en realidad una multiplicidad de historias y que narrarlas, a partir de diferentes fuentes, creaba planos de conexiones transversales que actuaban unos sobre otros, incluso confiriendo una imagen multifacética a la producción del vivir.

Desde esa perspectiva, no había un caso, sino muchas conexiones y líneas de sentido que se producían conforme se exponían los 
encuentros, que no eran solo "personificados", sino que también eran otros tipos de acontecimientos, de diversa naturaleza. Por ejemplo, un encuentro con una obra de arte puede disparar nuevas líneas de fuerzas de subjetivación, redefiniendo nuestros territorios existenciales y nuevas conexiones con los múltiples modos de vivir.

Sin embargo, se tornó un desafío poder observar los diversos casos-guías frente a la diversidad de encuentros expuestos, en los que se actualizaban todo el tiempo las conexiones y las relaciones de poder bajo la lente de la producción de acceso y de barreras, que para ese entonces ya era comprendida como accionamiento o inhibición de redes de conexiones existenciales.

Vale señalar que en esta construcción utilizamos de forma exploratoria el conceptoherramienta usuario-guía nómade, que desarrollamos durante este proyecto tomando como referencia los estudios de Merhy y Chakkour (18), Merhy y Franco (19) (sobre herramientas analizadoras y mapas analíticos), y los trabajos de Silva (14). En este proceso percibimos que la misma producción de la memoria, como herramienta constructora de los casos, era tan solo una entre muchas posibilidades de recortes y que eso requería de una problematización más clara sobre las reglas de enunciación de esos recortes narrativos, inscritos en las memorias producidas.

Para esa problematización, tomamos como ejemplo la necesidad de entender cómo las reglas de enunciación de la psiquiatría clásica imponen ciertos imaginarios y construcciones significantes sobre la locura como enfermedad, y los efectos que eso produce en los campos de fuerza de los procesos de subjetivaciones de los colectivos sociales. Por eso, buscamos crear nuestras propias enunciaciones y pensar sobre sus reglas, de modo que funcionaran como conceptos-herramientas que no fueran solo representaciones, sino también produjeran sentido en nuestras propias percepciones y afectos.

A continuación vamos a presentar algunos componentes de la construcción de esos conceptos-herramientas, procurando mostrar qué sentido tuvieron para nosotros en los diferentes momentos de la construcción de los "casos" estudiados.

\section{Redes de conexiones existenciales}

En el estudio del "caso Branca", corroborado por los demás usuarios-guías, percibimos que podíamos generar mapas de sus formas de existencias que no eran lineales y no tenían ninguna relación jerárquica en el diseño de sus modos de vivir, sino todo lo contrario. Muchas veces, esas formas de existencia eran disparadas en el mismo tiempo físico, lo que no implicaba el mismo tiempo lógico, obligándonos a construir esa imagen desde un campo de sentido singular, en el que ciertas relaciones y encuentros con otros -personas, servicios, establecimientos, eventos, etc.- tenían pertinencia, lo que nos permitía hablar de conexiones existenciales específicas. Aparecían varios tipos de redes en paralelo que producían sentido en sí mismas y que irrumpían en las otras, sin pedir permiso y sin mantener relaciones de subordinación o determinación entre ellas.

\section{Trayectorias de vida}

Como trabajamos con usuarios-guía nómades, nos dejamos llevar por sus "n-1" trayectos (fórmula de la multiplicidad), intentando recorrerlos y dibujarlos, dándoles registro visual. Percibimos que era posible conferir una línea de significación para ciertos trayectos, por ejemplo: trayecto como enfermo mental; como alumno de cierto curso; como trabajador de cierto tipo; como miembro de cierto grupo. No nos preocupamos en definir si estaban relacionados o no, si tenían o no líneas de determinaciones. No fue ese nuestro foco. Pretendíamos hablar de los "n-1" modos de producir caminos, persiguiéndolos en las redes en que se insertaban y constituían efectivamente, generando resultados, a veces imprevisibles, y con efectos en el campo de las relaciones de salud, pero sin posibilidad de ser considerados del orden del cuidado o protocolizados como ejes tecnológicos de acciones en salud. Cosas que tenían sentido para algunos usuarios-guías, eran justificaciones para el alejamiento de otros. 


\section{Historia de vida}

No es posible encontrar una linealidad en esta construcción, ni hay un único modo de traerla hacia el relato de la investigación. Por eso, tradujimos esa historia en muchas historias.

\section{Historia de los núcleos familiares}

Percibimos que una de las historias que producía muchos efectos en el mismo campo de cuidado, tenía que ver con la forma de construcción de las trayectorias en las redes de conexiones existenciales en lo que se podía reconocer como núcleo familiar. Por ejemplo, el modo como ciertos comportamientos de un joven fueron considerados "bizarros" y terminaron por llevarlo a una internación manicomial, con serias consecuencias para otras producciones de su existencia. Aunque no observamos líneas obligatorias de determinación, sí vimos la producción de efectos en los otros territorios.

Joaquim y Magda, dos de los usuariosguías estudiados, nos aportaron muchos elementos para comprender estos procesos. Al enmarcar sus situaciones en las narrativas de sus familiares, pudimos observar la construcción -también imaginaria- de que eran locos y como tal "merecían" cierto tipo de cuidado y observación. Ese lugar de la historia de los núcleos familiares, teniendo como eje ordenador el usuario-guía nómade, fue muy rico en nuestro material, pero requería comprender además lo que esos usuarios acarreaban como efecto de los encuentros en otro territorio no reconocido como familiar: el territorio de la vida común.

\section{Historia del territorio de la vida común}

Aquí destacamos todas las posibilidades de conexiones, de diferentes tipos y totalmente imprevisibles, que nos permitieron rescatar las historias de los usuarios-guías con otras personas, más allá de la familia. Por ejemplo, de niño Joaquim vivía en un área rural, tenía múltiples vínculos con otros que no pertenecían al núcleo familiar, otras actividades y encuentros no definidos a priori. Poder contar con algunas de esas historias en la construcción de las narrativas no es fácil dado que recurrir a las fuentes pasadas es casi imposible, pero afortunadamente en algunos casos todavía había vestigios de esa producción, a la que pudimos acceder. Ese territorio de la vida común es muy rico en efectos sobre el campo de la enfermedad y de la vida en general.

\section{Historia de las enfermedades}

En nuestros casos, estas historias fueron las de mayor riqueza narrativa, pues constituyen la centralidad producida en los modos de vivir de los usuarios a partir de las trayectorias construidas como "locos". Aquí las narrativas están muy marcadas por las reglas de enunciación que cruzan la construcción discursiva de las distintas fuentes, originadas institucionalmente en los servicios de salud. Es usual que el usuario sea reducido a un diagnóstico o a cierto estereotipo que construyen los servicios a través de los trabajadores y de las características institucionales. Por eso, el "Joaquim" de la época del manicomio es distinto del "Joaquim" de los servicios sustitutivos.

\section{Mapa de lo instituido a partir de las conexiones con los servicios de salud}

Este mapeo procuró dar visibilidad a los distintos tipos de servicios a los que estuvieron vinculados los casos y que los han instituido como usuarios. Se aproxima a la posibilidad de trabajar con la idea de itinerario terapéutico, pero como ese mapa es demarcado por lo estructurado, fue necesario invadirlo con otras narrativas, incluso las que actualizan las redes de cuidado, hechas por los cuidadores efectivos (que son diversos, de diferentes lugares, variando en el tiempo), para que se pueda traer a la escena la micropolítica del trabajo vivo en acto, desordenando la narrativa de lo instituido.

Redes de cuidado en acción: modos nómades del usuario-guía para moverse a través de sus múltiples modos de vivir

Ese lugar ha sido clave para avanzar tanto en la lógica cartográfica que se fue produciendo, como en los diversos trayectos y conexiones que ha posibilitado el territorio del cuidado. Su visibilidad se dio en la medida en que, por 
medio de las narrativas y registros, pudimos abordar la tensión entre la micropolítica del hacer en acto -construyendo la relación cuidador-cuidado- y el mundo del trabajo muerto, instituido en las normas y reglas de acción de las organizaciones e instituciones del cuidado en salud.

Por ejemplo, Magda, en distintos momentos se reencontró en las conexiones que logró producir y por las que fue producida, en el universo que la había atravesado en sus encuentros en un centro de atención psicosocial (CAPS), tanto con los demás usuarios, como con su equipo de referencia, o cuando se fue a vivir a una pensión y se relacionó con la propietaria. Construir ese mapeo con las informaciones de las fuentes ha sido fundamental.

\section{UN CONSTRUCTO INTENCIONAL DEL ESTUDIO: EL ALGORITMO “MEDIDOR" DE ACCESO Y BARRERAS}

Buscar indicadores de acceso y barreras es un esfuerzo clásico en el campo de la salud, generalmente limitado a la construcción de indicadores cuantitativos, como por ejemplo:

- Número de gestantes con cuidado prenatal en el primer trimestre del embarazo.

- Número de niños menores de un año con vacunación completa contra la polio.

- Número de usuarios esquizofrénicos que lograron consultas en un centro de atención psicosocial de su territorio.

- Número de portadores de catarata sometidos a cirugía correctiva en una determinada población y territorio.

- Número de pacientes de la red de cuidado en salud mental internados en manicomios en su región o fuera de ella.

- Número de pacientes con sufrimiento mental atendidos en los servicios de urgencia y emergencia que han sido inscritos en el centro de atención psicosocial de su territorio.

Son muchos los indicadores ya inventados para evaluar los servicios o las redes de cuidado. Todos son muy pertinentes, pero todos conllevan una marca: el acceso o la barrera como fenómeno casi material y físico de alguien "de afuera" que logra o no entrar dentro del servicio y obtener la posibilidad del cuidado, sea un servicio específico como un centro de atención psicosocial, una modalidad de cuidado, la internación o un taller terapéutico.

Pero esos indicadores no suelen revelar, como demostramos en este estudio, otras dimensiones fundamentales, dado que muchas veces "entrar" a una cierta red de cuidado puede ser el mejor camino para una barrera, desde el punto de vista de la construcción de un cuidado productor de "más vida" en los modos de existir de una persona.

Vemos aquí un reto complejo que queremos expresar en este artículo al mostrar cómo intentamos enfrentar el problema de construir indicadores de procesos que solo se pueden aprender cualitativamente. Así apostamos a la posibilidad de construir un "algoritmo", partiendo de una cuestión bien sencilla:

- Alguien que está siendo cuidado, ¿está ampliando o disminuyendo sus redes de conexiones existenciales?

- Si la respuesta es afirmativa, entonces hay producción de más vida.

- Si la respuesta es negativa, entonces es necesario reconocer que el acceso a ese cuidado está produciendo barreras para un vivir más calificado.

O sea, dependiendo del caso y del modo, el cuidado al que se ha accedido y está establecido puede funcionar como barrera para la producción de la vida. Entonces ese algoritmo puede indicar algo respecto de nuestro objeto de investigación. O mejor, creemos que indica cualitativamente, sin números, pero con fuerza expresiva la producción de modos de vivir más productores de vida en el campo existencial de alguien.

Por eso, repetimos: "se debe mirar acceso y barrera bajo la lógica de producir/inhibir conexiones existenciales", lo que podría indicarnos un producto innovador de este estudio.

Obtuvimos esa imagen a partir de la pobreza, o mejor dicho, de la miseria de las opciones producidas por las internaciones en instituciones cerradas. En el modo manicomial de cuidar, se genera más capacidad para producir 
muerte, que redes de conexiones de vida. $Y$, por lo tanto se producen barreras al cuidado.

Asimismo, en instituciones no cerradas como los centros de atención psicosocial o equivalentes, se pueden identificar situaciones en las que el mismo manejo clínico puede constituirse como barrera o al contrario.

Mirar todo proceso de cuidado instituido en los establecimientos o en los encuentros en general como generadores de efectos terapéuticos, desde el ángulo de la producción de identidades y de contractualización, abre para nosotros nuevos universos en la producción de conocimiento sobre el eje investigado.

Imaginamos que esa construcción puede adquirir la fuerza de un nuevo tipo de indicador para ser utilizado en las evaluaciones de las redes de cuidado en general y no solo en el campo de la salud mental.

\section{AGRADECIMIENTOS}

La construcción de este texto pertenece también a los trabajadores de la red de cuidado en salud mental del Servicio de Salud Dr. Cândido Ferreira, que han participado activamente en la investigación mencionada, la cual fue financiada por el Consejo Nacional de Desarrollo Científico y Tecnológico (CNPq) a través de la convocatoria No. 033/2008 del área de Salud Mental.

\section{REFERENCIAS BIBLIOGRÁFICAS}

1. Deleuze G, Guattari F. Mil Platôs: Capitalismo e Esquizofrenia. São Paulo: Editora 34; 2000. (vol. 1).

2. Merhy EE. Salud: cartografía del trabajo vivo. Buenos Aires: Lugar Editorial; 2006.

3. Merhy EE, Amaral H, organizadores. Reforma psiquiátrica no cotidiano II. São Paulo: Hucitec; 2007.

4. Basaglia F. A instituição negada. Rio de Janeiro: Graal; 2001.

5. Merhy EE. O conhecer militante do sujeito implicado: o desafio em reconhecê-lo como saber válido. En: Franco TB, et al. Acolher Chapecó. São Paulo: Hucitec; 2004.

6. Cecílio LCO. Modelos tecno-assistenciais em saúde: da pirâmide ao círculo, uma possibilidade a ser explorada. Cadernos de Saúde Pública. 1997;13(3):469-478.

7. Costa JF. História da psiquiatria no Brasil. Rio de Janeiro: Garamond; 2007.

8. Lancetti A. A clínica peripatética. São Paulo: Hucitec; 2006.

9. Franco TB. As redes na micropolítica do processo de trabalho em saúde. En: Pinheiro R,
Matos RA. Gestão em redes. Rio de Janeiro: LAPPIS-IMS, UERJ, ABRASCO; 2006.

10. Canguilhem G. O normal e o patológico. Rio de Janeiro: Forense Universitária; 1995.

11. Cecílio LCO, Merhy EE. A integralidade do cuidado como eixo da gestão hospitalar. En: Sentidos da integralidade. Rio de Janeiro: LAPPIS; 2003.

12. Feuerwerker LCM, Merhy EE. A contribuição da atenção domiciliar para a configuração de redes substitutivas de saúde: desinstitucionalização e transformação de práticas. Revista Panamericana de Salud Pública. 2008;24(3):180188.

13. Merhy EE, Feuerwerker LCM. Atenção domiciliar: medicalização e substitutividade [Internet]. Rio de Janeiro: Universidade Federal do Rio de Janeiro; 2007 [citado 12 nov 2011]. Disponible en: http://www.medicina.ufrj.br/micropolitica/pesquisas/atencaodomiciliar/textos/ad-medicalizacao _e_substitutividade.pdf

14. Silva E. Circo-teatro: Benjamim de Oliveira e a teatralidade circense no Brasil [Internet]. São Paulo: Editora Altana; 2007 [citado 10 nov 2011]. Disponible en: http://ufg.academia.edu/Robson Camargo/Books/298802/Circo-teatro_-_Benjamim _de_Oliveira_e_a_teatralidade_no_Brasil_de_Ermi nia_Silva_-_Download_Gratuito_FUNARTe 
15. Foucault M. A verdade e as formas jurídicas. Rio de Janeiro: NAU; 2002.

16. Deleuze G, Guattari F. Mil Platôs: Capitalismo e Esquizofrenia. São Paulo: Editora 34; 2000. (vol. 2).

17. Foucault M. Freud, Nietzsche e Marx. São Paulo: Princípio; 1997.

18. Merhy EE, Chakkour M. Em busca de ferramentas analisadoras das tecnologias em saúde: informação e o dia a dia de um serviço, interrogando e gerindo trabalho em saúde. En: Merhy EE, Onocko R, organizadores. Agir em Saúde: um desafio para o público. São Paulo: Hucitec; 1997. p. $85-112$.

19. Merhy EE, Franco TB. Mapas analíticos: una mirada sobre la organización y sus procesos de trabajo. Salud Colectiva. 2009;5(2):181-194.

\section{FORMA DE CITAR}

Merhy EE, Feuerwerker LCM, Silva E. Contribuciones metodológicas para estudiar la producción del cuidado en salud: aprendizajes a partir de una investigación sobre barreras y acceso en salud mental. Salud Colectiva. 2012;8(1):25-34.

Recibido el 10 de febrero de 2012

Versión final presentada el 11 de marzo de 2012

Aprobado el 21 de marzo de 2012 